\title{
THE CONVOLUTION TRANSFORMATION OF CERTAIN GENERALIZED FUNCTIONS AND ITS INVERSION ${ }^{1}$
}

BY A. H. ZEMANIAN

Communicated by F. John, March 21, 1966

1. We extend the convolution transformation [1]

$$
F(x)=\int_{-\infty}^{\infty} f(t) G(x-t) d t
$$

to certain classes of generalized functions. Since a variety of integral transformations are, through changes of variables, special cases of (1) $[1$, pp. 65-79], our present results yield a generalization and inversion theory for these integral transformations, many of which had not previously been extended to generalized functions.

The kernel $G$ is a probability frequency function of the type

$$
G(t)=\frac{1}{2 \pi i} \int_{-i \infty}^{i \infty} \frac{e^{s t}}{E(s)} d s
$$

where in this work $E(s)$ is restricted to the form

$$
E(s)=e^{b s} \prod_{k=1}^{\infty}\left(1-\frac{s}{a_{k}}\right) \exp \left(s / a_{k}\right),
$$

$b$ and $a_{k}$ being real numbers $\left(a_{k} \neq 0, a_{k} \rightarrow \infty\right)$, and $\sum a_{k}{ }^{2}<\infty . G(t)$ is said to be in Class I if there exist both positive and negative $a_{k}$, in Class II if all $a_{k}$ are positive and $\sum a_{k}^{-1}=\infty$, and in Class III if all $a_{k}$ are positive and $\sum a_{k}^{-1}<\infty$.

By a smooth function we mean a function that has continuous derivatives of all orders at all points of its domain.

2. Here we describe a theory that is suitable for every kernel $G$ whether in Class I, II, or III. Let $c$ and $d$ be two fixed real numbers, and let $\kappa_{c, d}(t)$ be a fixed smooth positive function such that

$$
\kappa_{c, d}(t)=\left\{\begin{array}{l}
e^{c t} \quad 1<t<\infty \\
e^{d t} \quad-\infty<t<-1 .
\end{array}\right.
$$

${ }^{1}$ This work was supported by the Air Force Cambridge Research Laboratories, Bedford, Mass., under contract AF19(628)-2981. 
$\mathscr{L}_{c, d}$ is the topological linear space of all complex-valued smooth functions $\phi(t)$ on $-\infty<t<\infty$ such that for each $k=0,1,2, \cdots$

$$
\begin{aligned}
\gamma_{k}(\phi) & =\max _{0 \leqq p \leqq t} \sup _{-\infty<t<\infty}\left|\kappa_{c, d}(t) \phi^{(p)}(t)\right|<\infty, \\
\phi^{(p)}(t) & \equiv \frac{d^{p} \phi}{d t^{p}},
\end{aligned}
$$

the topology of $\mathfrak{L}_{c, d}$ being that generated by the sequence of norms $\left\{\gamma_{k}\right\}_{k=1}^{\infty} \cdot \mathscr{L}_{c, d}$ is a sequentially complete countably normed space [2, p. 6].

For a given kernel $G(t)$, let

$$
\alpha_{1}=\max _{a_{k}<0}\left(a_{k},-\infty\right), \quad \alpha_{2}=\min _{a_{k}>0}\left(a_{k}, \infty\right) .
$$

For every fixed $x$ and nonnegative integer $k, G^{(k)}(x-t)$ as a function of $t$ is in $\mathfrak{S}_{c, d}$ if $c<\alpha_{2}$ and $d>\alpha_{1}$; this is apparent from the asymptotic behavior of $G(t)$ [1, pp. 108-109]. In this case, every member of the dual space $\mathfrak{L}_{c, d}^{\prime}$ of $\mathfrak{L}_{c, d}$ possesses a convolution transform, which we define as the application of $f \in \mathfrak{L}_{c, d}^{\prime}$ to $G(x-t) \in \mathscr{L}_{c, d}$ :

$$
F(x)=\langle f(t), G(x-t)\rangle \quad-\infty<x<\infty .
$$

Every distribution of bounded support is a member of $\mathscr{L}_{c, d}^{\prime}$, and every member of $\mathfrak{R}_{c, d}^{\prime}$ is a distribution on $-\infty<t<\infty$. Also, if $d<0<c$, then every tempered distribution is a member of $\mathfrak{L}_{c, d}^{\prime}$.

THEOREM 1. Given a kernel $G$, let $f \in \mathscr{L}_{c, d}^{\prime}$ where $c<\alpha_{2}$ and $d>\alpha_{1}$. Then, $F(x) \in \&_{a, b}$ where $a$ and $b$ are any real numbers such that $a<\min \left(-\alpha_{1},-c\right)$ and $b>\max \left(-\alpha_{2},-d\right)$. Moreover,

$$
F^{(k)}(x)=\left\langle f(t), G^{(k)}(x-t)\right\rangle \quad k=1,2,3, \cdots .
$$

To prove (3) we use an inductive argument, in which we consider

$$
\begin{gathered}
\frac{1}{\Delta x}\left[F^{(k-1)}(x+\Delta x)-F^{(k-1)}(x)\right]-\left\langle f(t), G^{(k)}(x-t)\right\rangle=\left\langle f(t), \theta_{\Delta x}(t)\right\rangle, \\
\theta_{\Delta x}(t)=\frac{1}{\Delta x}\left[G^{(k-1)}(x+\Delta x-t)-G^{(k-1))}(x-t)\right]-G^{(k)}(x-t)
\end{gathered}
$$

and prove that $\theta_{\Delta x}(t) \rightarrow 0$ in $\mathfrak{L}_{c, d}$ as $\Delta x \rightarrow 0$. Then, $F(x)$ is shown to be in $\mathscr{L}_{a, b}$ by invoking the following standard result: There exist a constant $C$ and an integer $r \geqq 0$ depending on $f$ such that

$$
\left|\kappa_{a, b}(x) F^{(k)}(x)\right| \leqq C \max _{0 \leqq \mu \leqq r} \sup _{t}\left|\kappa_{c, d}(t) \kappa_{a, b}(x) G^{(k+\mu)}(x-t)\right| .
$$


The right-hand side can be shown to be a bounded function of $x$ by exploiting the asymptotic behavior of $G(t)$ [1, pp. 108-109].

The real inversion formula of Hirschman and Widder [1, p. 128] can be extended to our generalized transformation (2) as follows. Let $\left\{b_{n}\right\}_{n=1}^{\infty}$ be a given sequence of real numbers such that $b_{n} \rightarrow 0$ as $n \rightarrow \infty$. With $D=d / d x$ and $a$ being a real number, let the operational symbol $e^{a D} F(x)$ denote $F(x+a)$. Set

$$
P_{n}(D)=\exp \left(\left(b-b_{n}\right) D\right) \prod_{k=1}^{n}\left(1-\frac{D}{a_{k}}\right) \exp \left(D / a_{k}\right) .
$$

THEOREM 2. Under the hypothesis of Theorem 1, let $F(x)$ be defined by (2). Then, in the sense of weak convergence in the space $D^{\prime}$ of Schwartz distributions [3],

$$
\lim _{n \rightarrow \infty} P_{n}(D) F=f .
$$

This is established by first showing that for any smooth function $\psi(x)$ of bounded support

$$
\begin{aligned}
\left\langle P_{n}(D) F, \psi\right\rangle & =\left\langle f(t),\left\langle\psi(x), P_{n}\left(D_{x}\right) G(x-t)\right\rangle\right\rangle, \\
D_{x} & =\frac{d}{d x}
\end{aligned}
$$

and then proving that $\left\langle\psi(x), P_{n}\left(D_{x}\right) G(x-t)\right\rangle \rightarrow \psi(t)$ in $\mathscr{L}_{c, d}$ as $n \rightarrow \infty$.

3. These results can be strengthened when the kernel $G$ is in either Class II or Class III. Indeed, the class of generalized functions, which possess convolution transforms (2), can be enlarged, but now (2) will exist in general only on an interval of the form $y_{f}<x<\infty$ where $y_{f}$ depends on $f$. Results similar to Theorems 1 and 2 can be established in these cases in essentially the same way as above.

\section{REFERENCES}

1. I. I. Hirschman and D. V. Widder, The convolution transform, Princeton Univ. Press, Princeton, N. J., 1955.

2. A. Friedman, Generalized functions and partial differential equations, PrenticeHall, Englewood Cliffs, N. J., 1963.

3. L. Schwartz, Théorie des distributions, Vols. I, II, Hermann, Paris, 1957, 1959.

State University of New York at Stony Brook 